DIVISION OF THE HUMANITIES AND SOCIAL SCIENCES

CALIFORNIA INSTITUTE OF TECHNOLOGY

PASADENA, CALIFORNIA 91125

COUNTING COMBINATORIAL CHOICE RULES

Federico Echenique

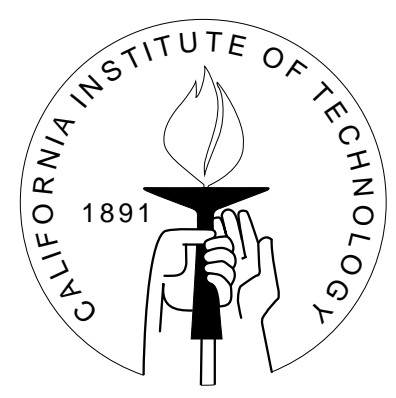

SOCIAL SCIENCE WORKING PAPER 1199

April 2004 


\title{
Counting Combinatorial Choice Rules
}

\author{
Federico Echenique
}

\begin{abstract}
I count the number of combinatorial choice rules that satisfy certain properties: KelsoCrawford substitutability, and independence of irrelevant alternatives. The results are important for two-sided matching theory, where agents are modeled by combinatorial choice rules with these properties. The rules are a small, and asymtotically vanishing, fraction of all choice rules. But they are still exponentially more than the preference relations over individual agents - which has positive implications for the Gale-Shapley algorithm of matching theory.
\end{abstract}

JEL classification numbers: C65,C78

Key words: Substitutability, Choice rules, Matching markets, Gale-Shapley Algorithm 


\title{
Counting Combinatorial Choice Rules *
}

\author{
Federico Echenique
}

\section{Introduction}

Consider hiring a team of workers, from a set $A$ of available workers. The decision of hiring worker $x$ is not independent of the decision to hire worker $y$; the workers may be complements or substitutes. Let $C(A) \subseteq A$ be the workers hired. The function $C$ is called a (combinatorial) choice rule. I shall give results on the number of functions $C$ that satisfy various properties.

The main application I have in mind is the theory of matching markets (Roth and Sotomayor, 1990). In many-to-one, and many-to-many, matching theory, some agents are matched to a set of agents. These agents' behavior is modeled as a choice rule.

The classical results on matching markets - among others, that the core is nonemptyrequire structure on the choice rules: substitutability and independence of irrelevant alternatives (IIA). Substitutability was introduced, and applied to matching markets, by Kelso and Crawford (1982). IIA is a rationality assumption. Further, substitutability and IIA is the only structure needed (this is clear from Blair (1988), who first proved some of the classical results).

I calculate the number of choice rules that satisfy substitutability, and substitutability and IIA. The main implications of the results are:

1. The choice rules that satisfy substitutability are a small, and asymptotically vanishing, fraction of all choice rules. Arguably, then, substitutability is a strong assumption. In continuous models, one routinely disregards cases with Lebesguemeasure zero; the same logic suggests that substitutability is a strong assumption. The obvious caveat is: Even if they are scarce, the substitutable choice rules may nevertheless often occur. For example, because they are induced by certain behaviors - such as "responsive" preferences (Roth and Sotomayor, 1990, page 173).

\footnotetext{
*I am very grateful to Ilya Segal, for posing this problem, and for discussions on the results.
} 
2. The choice rules that satisfy substitutability and IIA are exponentially more than the preference relations over individual workers. So the choice rules with the right structure are small, ma non troppo.

This result has an important implication for the Gale-Shapley algorithm for finding a matching in the core. Segal (2003) proves that the Gale-Shapley algorithm requires approximately as much communication as communicating a preference relation over individual workers. My results and Segal's then imply that the algorithm requires exponentially less communication than full revelation of agents' choice rules. The implication helps explain why the Gale-Shapley algorithm is so widely used in practice.

See Segal (2003) on what communication means, and why full revelation is the right benchmark.

My results exploit a connection between choice rules and a well-studied, but unsolved, problem in combinatorics: The problem of counting the monotone boolean functions, called "Dedekind's problem." I show that Dedekind's problem is equivalent to the problem of counting substitutable choice rules. And that Dedekind's problem gives bounds on the substitutable and IIA choice rules. Then, existing bounds and approximations to Dedekind's problem give bounds and approximations to the problem of counting choice rules.

The numbers involved are surprising. Suppose 8 objects can be chosen; much fewer than in actual matching markets. Already with 8 objects, the substitutable choice rules are a small fraction of the number of choice rules. There are $1.8 \times 10^{308}$, roughly a centillion, different choice rules (more choice rules than protons in the universe). The number of substitutable choice rules is of the order $10^{99}$.

It may be easier to think of bits: With 8 objects, it takes one kilobit (1028 bits) to communicate one choice rule, while communicating a substitutable rule takes 329 bits. Communicating a substitutable and IIA choice rule takes between 35 and 285 bits. And communicating a preference relation over individual objects takes 23 bits. ${ }^{1}$

Non-combinatorial choice rules. Consider non-combinatorial choice: the objects of choice are mutually excluding, and so choosing $x$ is independent of the decision to choose $y$. There is an extensive theory of non-combinatorial choice rules in decision theory and social choice (see, for example, Moulin (1991) or Austen-Smith and Banks (1998)).

Now, $C(A)$ may still have more than one element. The interpretation is that any of the elements in $C(A)$ could be chosen. The model is identical to the model in combinatorial choice. But the structure has a very different interpretation.

My calculations for combinatorial choice rules have some obvious, but possibly interesting, implications for non-combinatorial choice rules.

\footnotetext{
${ }^{1}$ I explain these calculations in Section 4.6.
} 
Substitutability is formally identical to the condition called (Sen's) $\alpha$ (or Chernoff's condition) in the non-combinatorial model. Thus, the choice rules that satisfy $\alpha$ vanish as a proportion of all choice rules.

I also show that the choice rules that satisfy $\alpha$ are exponentially more than the choice rules that are rationalizable by a preference relation. And that there are more rules that satisfy (Sen's) $\beta$ than there are rules that satisfy $\alpha$. So there are also many more rules that satisfy $\beta$ than there are rationalizable rules.

Section 2 presents the results on combinatorial choice rules. The proof of the main theorem is deferred to Section 4. Section 3 presents the results on non-combinatorial choice rules.

\section{Combinatorial Choice Rules}

\subsection{The Model}

Let $X$ be a finite set of cardinality $n$. Without loss, let $X=\{1, \ldots n\}$. A choice rule is a map $C: 2^{X} \rightarrow 2^{X}$ such $C(A) \subseteq A$ for all $A \subseteq X$. Let $\mathcal{C}(n)$ be the set of all choice rules, i.e.

$$
\mathcal{C}(n)=\Pi_{A \subseteq X} 2^{A}
$$

For each choice rule $C$ there is an associated choice rule $R$, defined by $R(A)=$ $A \backslash C(A)$. If $C(A)$ are the chosen elements of $A, R(A)$ are the rejected elements.

A choice rule $C$ satisfies substitutability if, for all $A, B \subseteq X$,

$$
A \subseteq B \text { implies } C(B) \cap A \subseteq C(A) .
$$

Equivalently, $C$ satisfies substitutability if $A \subseteq B$ implies $R(A) \subseteq R(B)$.

A choice rule $C$ satisfies independence of irrelevant alternatives (IIA) if, for every $A, B, \subseteq X$, if $C(B) \subseteq A \subseteq B$ then $C(B)=C(A)$.

The theory of matching markets requires two hypotheses on choice: substitutability and IIA (see Kelso and Crawford (1982), Blair (1988) and Roth and Sotomayor (1990)).

\section{$2.2 \quad$ Results}

The calculation of $|\mathcal{C}(n)|$ is very simple, and probably known (I could not find a reference in print). I include it for use in the rest of the paper.

Theorem 1. $|\mathcal{C}(n)|=2^{n 2^{n-1}}$ 
Proof. From the definition of $\mathcal{C}(n)$,

$$
|\mathcal{C}(n)|=\prod_{k=1}^{n} 2^{k\left(\begin{array}{l}
n \\
k
\end{array}\right)} .
$$

So,

$$
\begin{aligned}
\log |\mathcal{C}(n)| & =\sum_{k=1}^{n} k\left(\begin{array}{l}
n \\
k
\end{array}\right) \\
& =\sum_{k^{\prime}=0}^{n-1} n \frac{(n-1) !}{\left(n-k^{\prime}-1\right) ! k^{\prime} !} \\
& =n 2^{n-1} .
\end{aligned}
$$

Thus $|\mathcal{C}(n)|=2^{n 2^{n-1}}$.

Let $\mathcal{C}_{1}(n)$ be the set of choice rules that satisfy substitutability. Let $\mathcal{C}_{2}(n)$ be the set of choice rules that satisfy substitutability and IIA; $\mathcal{C}_{2}(n)$ is the important class of rules for the theory of matching markets. If agents' choice rules are in $\mathcal{C}_{2}(n)$, the classical results on matching markets follow. And all choice rules in $\mathcal{C}_{2}(n)$ are rationalizable by a preference relation over subsets of $X .^{2}$

The theorem employs the following notation. Let $S(K, k)$ denote the number of partitions of $\{1, \ldots K\}$ into $k$ sets. $S(K, k)$ is called the Stirling number of the second kind (see e.g. Comtet (1974)). All logarithms in the paper are base 2, unless denoted by ln, in which case the base is $e$.

\section{Theorem 2.}

$$
\begin{aligned}
& 2^{n\left(\begin{array}{c}
n-1 \\
\lfloor(n-1) / 2\rfloor
\end{array}\right)} \leq\left|\mathcal{C}_{1}(n)\right| \leq 2^{(n+\kappa \log (n))\left(\begin{array}{c}
n \\
n / 2
\end{array}\right)} \\
& 2^{\left(\begin{array}{c}
n-1 \\
(n-1) / 2\rfloor
\end{array}\right)} \leq\left|\mathcal{C}_{2}(n)\right|, \\
&\left|\mathcal{C}_{2}(n)\right| \leq \sum_{0 \leq k \leq K \leq n}\left(\begin{array}{c}
n \\
K
\end{array}\right)\left(\begin{array}{c}
K \\
k
\end{array}\right) 2^{(1+\kappa \log (n) / n)\left(\begin{array}{c}
n \\
n / 2
\end{array}\right)} k ! S\left(\left(\begin{array}{c}
K \\
\lfloor K / 2\rfloor
\end{array}\right), k\right),
\end{aligned}
$$

and $\log \left|\mathcal{C}_{1}(n)\right| \sim n\left(\begin{array}{c}n-1 \\ \lfloor(n-1) / 2\rfloor\end{array}\right)$. $\kappa$ is a constant.

The proof of Theorem 2 is in Section 4. Section 4.1, in particular, presents the main insight behind the proof.

I bring out the implications of Theorem 2 in Corollaries 3 and 5 . The implications were discussed in the Introduction. The corollaries do not use the upper bound on $\left|\mathcal{C}_{2}(n)\right|$ from Theorem 2. I use the upper bound in my calculations (Section 4.6), but I do not know if it gives an asymptotic improvement over the upper bound on $\left|\mathcal{C}_{1}(n)\right|$.

Corollary 3. $\left|\mathcal{C}_{1}(n)\right| /|\mathcal{C}(n)|$ is $o\left(2^{-2^{n}}\right)$, and $\log \left|\mathcal{C}_{1}(n)\right| / \log |\mathcal{C}(n)|$ is o (1).

\footnotetext{
${ }^{2}$ In fact, any $C \in \mathcal{C}_{2}(n)$ is rationalizable by any linear extension of the order $A P B$ if $A=C(A \cup B)$ the order used by Blair (1988). I thank Chris Chambers for pointing this out.
} 
Proof. Using the upper bound on $\left|C_{1}\right|$ from Theorem 2, and Stirling's formula applied to $\left(\begin{array}{c}n \\ \lfloor n / 2\rfloor\end{array}\right)$,

$$
\begin{aligned}
\log \frac{2^{2^{n}}\left|\mathcal{C}_{1}(n)\right|}{|\mathcal{C}(n)|} & \leq(n+\kappa \log (n))\left(\begin{array}{c}
n \\
\lfloor n / 2\rfloor
\end{array}\right)+2^{n}-n 2^{n-1} \\
& \sim\{(\sqrt{n}+(\kappa / \sqrt{n}) \log (n)) \sqrt{2 / \pi}+1-n / 2\} 2^{n} .
\end{aligned}
$$

The term in brackets goes to $-\infty$, so $\frac{2^{2^{n}}\left|\mathcal{C}_{1}(n)\right|}{|\mathcal{C}(n)|} \rightarrow 0$, as $n \rightarrow \infty$. Thus $\left|\mathcal{C}_{1}(n)\right| /|\mathcal{C}(n)|$ is $o\left(2^{-2^{n}}\right)$.

By a similar calculation,

$$
\begin{aligned}
\frac{\log \left|\mathcal{C}_{1}(n)\right|}{\log |\mathcal{C}(n)|} & \leq \frac{(n+\kappa \log n)\left(\begin{array}{c}
n \\
\lfloor n / 2\rfloor
\end{array}\right)}{n 2^{n-1}} \\
& \sim\left(1 / \sqrt{n}+\frac{\kappa \log n}{n^{3 / 2}}\right) \frac{2^{3 / 2}}{\sqrt{\pi}}
\end{aligned}
$$

So $\log \left|\mathcal{C}_{1}(n)\right| / \log |\mathcal{C}(n)|$ is $o(1)$.

A preference relation over $X$ is a transitive binary relation $\succ$ over $X$. Let $\mathcal{P}(n)$ be the set of all preference relations over $X$. The following theorem is due to Gross (1962) (Barthélémy (1980) rediscovered the theorem, and presents a different proof).

Theorem 4. $\mathcal{P}(n) \sim \frac{n !}{2(\ln 2)^{n+1}}$

Corollary 5. $\log |\mathcal{P}(n)| / \log \left|\mathcal{C}_{2}(n)\right|$ is $o\left(n^{2} 2^{-n}\right)$

Proof. Using the lower bound on $\left|\mathcal{C}_{2}(n)\right|$ from Theorem 2,

$$
\frac{\log |\mathcal{P}(n)|}{\log \left|\mathcal{C}_{2}(n)\right|} \leq \frac{\log |\mathcal{P}(n)|}{\left(\begin{array}{c}
n-1 \\
\lfloor(n-1) / 2\rfloor
\end{array}\right)}=h(n) \text {. }
$$

Using Theorem 4,

$$
h(n) \sim \frac{\log n !-(n+1) \log (\ln 2)-1}{2^{n}(n \pi / 2)^{-1 / 2}} .
$$

The result now follows from $\log n ! \sim n \log n$, so $(\sqrt{n} \log n !) / n^{2} \rightarrow 0$.

\section{Non-combinatorial Choice Rules}

\subsection{The Model}

The model is formally the same as in Section 2.1: given a set $X$ of alternatives, a choice rule is a function $C: 2^{X} \rightarrow 2^{X}$ with $C(A) \subseteq A$, for all $A \subseteq X$. But now the elements 
of $X$ are mutually exclusive choices. Still, $C(A)$ may not be a singleton because the decision maker is happy choosing any of the elements of $C(A)$. Until now, $C(A)$ may not be a singleton because the decision maker decides to choose, as a package, $C(A)$ over any other subset of $A$.

Non-combinatorial choice rules have been studied extensively in the literature on individual and social choice (see e.g. Moulin (1991), or Austen-Smith and Banks (1998)). My model differs in one aspect from the standard model of choice: I allow that $C(A)=$ $\emptyset$. Some researchers allow $C(A)=\emptyset$ (Aizerman and Aleskerov, 1995). But the most commonly used model rules out $C(A)=\emptyset$.

There are four advantages to allowing $C(A)=\emptyset$. First, since $\emptyset$ is always a feasible choice, it allows one to model either indecision, or the presence of an outside option. Second, it gives a symmetric treatment of a choice rule $C$, and its associated $R$. The advantage of a symmetric treatment will be clear from Proposition 6 . Third, while it implies some minor changes in the results on non-combinatorial choice (See Appendix A), it does not seem to violate the spirit of the results on non-combinatorial choice. The non-empty-valued $C$ remain a special case. Finally, of course, the model coincides with combinatorial choice.

\subsection{Results}

When applied to non-combinatorial choice rules, Statement 1 is not called substitutability. It is called Sen's $\alpha$ (or Chernoff's condition-for Chernoff (1954), who introduced it). The difference is more subtle than may seem at first; it is not just a matter of terminology: Statement 1 is not what $\alpha$ amounts to in the combinatorial interpretation of $C$. In fact, $\alpha$ amounts to IIA.

Now, knowing that Sen's $\alpha$ coincides with substitutability, one obtains the obvious restatements of the results in Section 2.2 to the $\alpha$ property in non-combinatorial choice. I do not include the restatements in the paper.

A choice rule $C$ satisfies Sen's $\beta$ if, for all $A, B \subseteq X, A \subseteq B$ and $C(A) \cap C(B) \neq \emptyset$ imply that $C(A) \subseteq C(B)$. The following proposition is trivial; $R$ is the rejection choice rule associated with $C$.

Proposition 6. If $R$ satisfies substitutability, then $C$ satisfies $\beta$.

Proposition 6 implies that there are more choice rules that satisfy $\beta$ than choice rules that satisfy $\alpha$. So the results in Section 2.2 give also a lower bound on the choice rules that satisfy $\beta$.

Let $C$ be a choice rule. Say that a preference relation $\succ$ over $X \cup\{\emptyset\}$ rationalizes $C$ if, for all $A \subseteq X, x \in C(A)$ if and only if $x \succ \emptyset$ and $y \nsucc x$, for all $y \in A$. Let $\mathcal{C}_{3}(n)$ be the set of rules that are rationalized by some preference relation. 
Theorem 7. $\left|\mathcal{C}_{3}(n)\right| \sim \frac{n !}{(\ln 2)^{n+1}}$.

The proof of Theorem 7 is similar to Gross's (1962) proof of Theorem 4. But Theorem 4 is not immediately applicable to $\left|\mathcal{C}_{3}(n)\right|$; since $\emptyset$ is an option, $\mathcal{C}_{3}(n)$ contains strictly more elements than $\mathcal{P}(n)(n)$ (or $\mathcal{P}(n)(n+1))$.

Proof. Preference relations $\succ$ and $\succ^{\prime}$ over $X \cup\{\emptyset\}$ give rise to the same $C$ if and only if

1. $\{x \in X: x \succ \emptyset\}=\left\{x \in X: x \succ^{\prime} \emptyset\right\}$, and

2. they coincide on all pairs $(x, y) \in X^{2}$ such that $x \succ \emptyset$ and $y \succ \emptyset$.

So there are as many $C$ in $\mathcal{C}_{3}(n)$ as classes of preference relations that differ in either (1) or (2). These can be counted as follows: First choose $k+1=1 \ldots n+1$, the rank of $\emptyset$ in the rationalizing $\succ$. Second, choose the set of $k$ elements of $X$ that is ranked above $\emptyset$. Third, choose a preference relation over the elements that are ranked above $\emptyset$.

Let $w_{k}=|\mathcal{P}(n)(k)|$ be the number of preference relations over $\{1, \ldots k\}$. Then $\left|\mathcal{C}_{3}(n)\right|=\sum_{k=0}^{n}\left(\begin{array}{l}n \\ k\end{array}\right) w_{k}$.

Gross (1962) shows that $w_{k}=\frac{1}{2} \sum_{i=0}^{\infty}\left(\begin{array}{c}k \\ i\end{array}\right) 2^{-i}$. So,

$$
\begin{aligned}
\left|\mathcal{C}_{3}(n)\right| & =\frac{1}{2} \sum_{i=0}^{\infty} 2^{-i} \sum_{k=0}^{n}\left(\begin{array}{l}
n \\
k
\end{array}\right) i^{k} \\
& =\frac{1}{2} \sum_{i=0}^{\infty} 2^{-i}(1+i)^{n} .
\end{aligned}
$$

Now I estimate the series above by $1 / 2 \int_{0}^{\infty} 2^{-x}(1+x)^{n} d x$, since the difference between $\sum_{i=0}^{\infty} 2^{-i}(1+i)^{n}$ and $\sum_{i=1}^{\infty} 2^{-i}(1+i)^{n}$ is negligible when $n$ is large.

$$
\begin{aligned}
1 / 2 \int_{0}^{\infty} 2^{-x}(1+x)^{n} d x & =\int_{0}^{\infty} 2^{-y} y^{n} d y-\int_{0}^{1} 2^{-y} y^{n} d y \\
& =\left(\frac{1}{\ln 2}\right)^{n} \int_{0}^{\infty} z^{n} e^{-z} \frac{d z}{\ln 2}-\int_{0}^{1} 2^{-y} y^{n} d y \\
& =\frac{n !}{(\ln 2)^{n+1}}-\int_{0}^{1} 2^{-y} y^{n} d y
\end{aligned}
$$

The first equality follows from the change of variables $y=x+1$, and from adding and subtracting the integral from 0 to 1 . The second equality follows from the change of variable $z=y \ln 2$. The third equality follows from recognizing the Gamma function, and using $\Gamma(n+1)=n$ !. Since $\int_{0}^{1} 2^{-y} y^{n} d y \rightarrow 0,\left|\mathcal{C}_{3}(n)\right| \sim \frac{n !}{(\ln 2)^{n+1}}$.

In light of Theorem 7, the following corollary follows just like Corollary 5. 
Corollary 8. $\log \left|\mathcal{C}_{3}(n)\right| / \log \left|\mathcal{C}_{2}(n)\right|$ is o $\left(n^{2} 2^{-n}\right)$.

And, for $\alpha$ alone,

Corollary 9. $\left|\mathcal{C}_{3}(n)\right| /\left|\mathcal{C}_{1}(n)\right|$ is $o\left(2^{-2^{n}}\right)$

Proof. By the proof of Theorem 7, $\left|\mathcal{C}_{3}(n)\right|=\sum_{k=0}^{n}\left(\begin{array}{l}n \\ k\end{array}\right) w_{k} \leq n\left(\begin{array}{c}n \\ \lfloor n / 2\rfloor\end{array}\right) w_{n}$. So, using the lower bound on $\mathcal{C}_{1}(n)$ from Theorem 2 ,

$$
\frac{\left|\mathcal{C}_{1}(n)\right|}{2^{2^{n}}\left|\mathcal{C}_{3}(n)\right|} \geq \frac{2^{n\left(\begin{array}{c}
n-1 \\
\lfloor(n-1) / 2\rfloor
\end{array}\right)}}{2^{2^{n}} n\left(\begin{array}{c}
n \\
\lfloor n / 2\rfloor
\end{array}\right) w_{n}} \sim h(n)=\frac{2^{\frac{n}{\sqrt{n-1}} 2^{n-1} \sqrt{2 / \pi}}}{2^{2^{n}} \sqrt{n} 2^{n} \sqrt{2 / \pi} \frac{n !}{2(\ln 2)^{n+1}}} .
$$

Where I have used Stirling's formula to estimate the $\left(\begin{array}{c}n \\ \lfloor n / 2\rfloor\end{array}\right)$ terms, and Theorem 4 for $w_{n}$.

In turn, and using log to simplify the expression,

$$
\log h(n)=\frac{n 2^{n}}{\sqrt{(n-1) 2 \pi}}-2^{n}-(1 / 2) \log n-n-\log n !+(n+1) \log (\ln 2)+\Omega,
$$

where $\Omega=2-(1 / 2) \log (2 / \pi)$

Since $\log n !$ is $o\left(n^{2}\right), \log h(n) \rightarrow \infty$.

\section{Proof of Theorem 2}

\subsection{Idea of the proof, and auxiliary definitions.}

A collection $a \subseteq 2^{X}$ of subsets of $X$ is an antichain if $A, B \in a$ implies $A \nsubseteq B$; antichains are also called Sperner families. A collection $a \subseteq 2^{X}$ of subsets of $X$ is an (order) filter if $A \in a$ and $A \subseteq B$ implies $B \in a$.

The number of antichains is equivalent to Dedekind's problem: finding the number of functions $f: 2^{X} \rightarrow\{0,1\}$ that are monotone. The equivalence between the number of antichains and Dedekind's problem is easy to show (see e.g. Engel (1997)); the argument is similar to that of the proof of Lemma 10 below. Dedekind's problem is an open problem. But there are known bounds, and asymptotic formulas.

The proof of Theorem 2 proceeds by relating the choice rules in $\mathcal{C}_{1}(n)$ and $\mathcal{C}_{2}(n)$ $\left(\mathcal{C}_{1}(n)\right.$ is easy, $\mathcal{C}_{2}(n)$ takes much more work) to collections of antichains, and thus to Dedekind's problem. The literature on Dedekind's problem then provides the bounds and asymptotic formulas reported in Theorem 2.

If $C$ is a substitutable rule, rejection is monotone: if $x$ is rejected from $A$, then $x$ is rejected from $B$, for all $B \supseteq A$. Let $T_{x}$ be the collection of sets from which $x$ is rejected, 
so $T_{x}$ is a filter, and $T_{x}$ is characterized by its minimal elements. The minimal elements of $T_{x}$ form an antichain.

Let $\left(T_{x}: x \in X\right)$ be a list of such filters. I shall work with $\left(T_{x}: x \in X\right)$, instead of $C$. Thus one characterizes the substitutable $C$ as assignments of an antichain to each element of $X$. The characterization allows me to use results on Dedekind's problem.

\subsection{Results on Filters}

I now present a series of short lemmas on filters. The results give some necessary, and some sufficient, conditions for a system of filters to correspond to a choice rule that satisfies substitutability and IIA. But the results fall short of a characterization of the substitutable and IIA choice rules.

A filter at $x$ is a filter $T_{x}$ such that $x \in A$, for all $A \in T_{x}$.

Lemma 10. We can identify each substitutable $C$ with a collection of filters $\left(T_{x}: x \in X\right)$, such that $T_{x}$ is a filter at $x$, for all $x \in X$. The isomorphism between $C$ and $\left(T_{x}\right)$ is: Given $C$, let $\left(T_{x}\right)$ be

$$
T_{x}=\{A \subseteq X: x \in A \backslash C(A)\}
$$

And given $\left(T_{x}\right)$ let

$$
C(A)=\left\{x \in A: A \notin T_{x}\right\}
$$

Proof. Identify $C$ with $R$, defined as $R(A)=A \backslash C(A)$. $C$ is substitutable if and only if $R$ is monotone. First, the collection $\left(T_{x}\right)$ defined as above is a collection of filters. To see this, note that $x$ is in all its elements by definition. Also, if $A \in T_{x}$ and $A \subseteq B$, then $x \in R(A) \subseteq R(B)$ by monotonicity of $R$, and $R(B) \subseteq B$, so $B \in T_{x}$.

Second, for each filter $\left(T_{x}: x \in X\right)$, define $R$ by $R(A)=\left\{x \in X: A \in T_{x}\right\}$. Then $R(A) \subseteq A$ since $\left(A \in T_{x} \Rightarrow x \in A\right)$. And $R$ is monotone since $T_{x}$ is a filter.

Now fix a collection of filters $\left(T_{x}: x \in X\right)$.

Lemma 11. $\left(T_{x}\right)$ satisfies IIA if and only if, for every $A, B \subseteq X$ with $A \subseteq B$, and every $x \in A$ :

$$
\left((\forall z \in B \backslash A)\left(B \in T_{z}\right)\right) \wedge\left(B \in T_{x}\right) \Rightarrow\left(A \in T_{x}\right)
$$

Proof. Let $A, B \subseteq X$ with $A \subseteq B$ and $C(B) \subseteq A$. By substitutability, $A \backslash C(A) \subseteq$ $B \backslash C(B)$. Since $A \subseteq B, A \backslash C(A) \subseteq A \backslash C(B)$.

Note that, since $C(B) \subseteq B$, the statement $\left[(\forall z \in B \backslash A)\left(B \in T_{z}\right)\right]$ is equivalent to $C(B) \subseteq A$.

(Sufficiency) $C(B) \subseteq A$ and $B \in T_{x}$ imply $A \in T_{x}$. Thus $A \backslash C(A) \supseteq A \backslash C(B)$. So $A \backslash C(A)=A \backslash C(B)$. This shows that $C(A)=C(B)$. 
(Necessity) $\left[(\forall z \in B \backslash A)\left(B \in T_{z}\right)\right]$ is $C(B) \subseteq A$. So IIA implies $A \backslash C(A)=A \backslash C(B)$. Hence, for all $x \in A$, if $B \in T_{x}$ then $A \in T_{x}$.

For each $B \subseteq X$, let $Z^{B}=\left\{z \in B: B \in T_{z}\right\}$.

Lemma 12. ( $\left.T_{x}\right)$ satisfies IIA if and only if, for every $B \subseteq X$, for every $z \in Z^{B}$,

$$
\left(B \backslash Z^{B}\right) \cup\{z\} \in T_{z}
$$

Proof. (Necessity) Let $B \subseteq X$. Let $z \in Z^{B}$. Let $A=\left(B \backslash Z^{B}\right) \cup\{z\}$. Then for every $x \in B \backslash A, B \in T_{x}$. Further $B \in T_{z}$ so IIA, by Lemma 11, implies $A \in T_{z}$.

(Sufficiency) Let $A \subseteq B \subseteq X$ be such that, for all $x \in B \backslash A, B \in T_{x}$. Then $B \backslash A \subseteq Z^{B}$. Let $z \in A$ be such that $B \in T_{z}$. By hypothesis, $\left(B \backslash Z^{B}\right) \cup\{z\} \in T_{z}$. But $B \backslash A \subseteq Z^{B}$ implies that $\left(B \backslash Z^{B}\right) \cup\{z\} \subseteq A$. Then $A \in T_{z}$ since $T_{z}$ is a filter. Hence, by Lemma $11,\left(T_{x}\right)$ satisfies IIA.

Given $\left(T_{x}: x \in X\right)$, for each $x$, let $M_{x}$ be the collection of minimal elements of $T_{x}$. So,

$$
M_{x}=\left\{B \in T_{x}:(A \subseteq B) \wedge\left(A \in T_{x}\right) \Rightarrow A=B\right\} .
$$

Lemma 13. If $\left(T_{x}\right)$ satisfies IIA, then, for every $x \in X$ and every $B \in M_{x},\{x\}=Z^{B}$.

Proof. IIA implies (Lemma 12) that $\left(B \backslash Z^{B}\right) \cup\{x\} \in T_{x}$. Then $\left(B \backslash Z^{B}\right) \cup\{x\} \subseteq B$ implies that $\left(B \backslash Z^{B}\right) \cup\{x\}=B$. So $\{x\}=Z^{B}$.

Lemma 14. Let $\left(T_{x}\right)$ satisfy IIA. For every $x, y \in X$, if $x \neq y$ then $M_{x} \cap M_{y}=\emptyset$.

Proof. Immediate from Lemma 13.

Lemma 15. If $\left(T_{x}\right)$ satisfies IIA, then, $\cup\left\{M_{x}: x \in X\right\}$ is an antichain.

Proof. Let $A \in M_{x}, B \in M_{y}$ with $A \neq B$. If $x=y$ then $A \nsubseteq B$ since $M_{x}$ is the collection of minimal sets in $T_{x}$. If $x \neq y$ then $A \subseteq B$ would imply that $B \in T_{x}$, as $A \in T_{x}$ and $T_{x}$ is a filter. But then $x \in Z^{B}$. Impossible, as IIA implies that $Z^{B}=\{y\}$ by Lemma 13 .

Remark 16. By Lemmas 14 and 15, $\left\{M_{x}: x \in X\right\}$ is a partition of some antichain.

Lemma 17. Let $\left(T_{x}: x \in X\right)$ satisfy IIA. For all $x \in X$, one the following must be true:

- $M_{x}=\emptyset$

- $M_{x}=\{\{x\}\}$

- $\exists A \in M_{x}$ such that $(\forall y \in A \backslash\{x\})\left(M_{y}=\emptyset\right)$. 
Proof. Let $M_{x} \neq \emptyset$, and $M_{x} \neq\{\{x\}\}$, so $\{x\} \notin M_{x}$. We need to prove that $\exists A \in M_{x}$ such that $\forall y \in A \backslash\{x\}, M_{y}=\emptyset$.

Suppose, by way of contradiction, that for every $A \in M_{x}$, there is $y \in A$ with $y \neq x$ such that $T_{y} \neq \emptyset$.

Pick $A \in M_{x}$, let $y_{1} \in A$ and $D_{1} \in M_{y_{1}}$. Let $B_{1}=A \cup D_{1}$, so $x, y_{1} \in Z^{B_{1}}$. By IIA and Lemma 12 there is $A^{\prime} \in M_{x}$ with $A^{\prime} \subseteq\left(B_{1} \backslash Z^{B_{1}}\right) \cup\{x\}$. Note $y_{1} \notin A^{\prime}$. By our assumption, there is $y_{2} \in A^{\prime}$ with $M_{y_{1}} \neq \emptyset$. Let $D_{2} \in M_{y_{2}}$. Note $y_{1} \neq y_{2}$.

Given a sequence of different elements $y_{1}, y_{2}, \ldots y_{k-1}, k \geq 2$, and corresponding sets $D_{1}, D_{2}, \ldots D_{k-1}$ with $D_{l} \in M_{y_{l}}$, with $1 \leq l \leq k-1$. Let

$$
B_{k}=A \cup\left(\cup_{l=1}^{k-1} D_{l}\right) .
$$

So $x \in Z^{B_{k}}$ and $y_{l} \in Z^{B_{l}}, 1 \leq l \leq k-1$. By IIA and Lemma 12 there is $A^{\prime} \in M_{x}$ with $A^{\prime} \subseteq\left(B_{k} \backslash Z^{B_{k}}\right) \cup\{x\}$. By hypothesis, there is $y_{k} \in A^{\prime}$ with $T_{y_{k}} \neq \emptyset$. so there is a corresponding $D_{k} \in M_{y_{k}}$. Since $y_{k} \in A^{\prime}, y_{k} \neq y_{l}, 1 \leq l \leq k-1$.

But $X$ is a finite set, so this has to stop, and there must be a $A \in M_{x}$ with $T_{y}=\emptyset$ for all $y \in A$.

Lemma 18. Let $\left(T_{x}: x \in X\right)$ satisfy IIA. Then, for all $x \in X$,

$$
\cap\left\{A: A \in M_{x}\right\} \subseteq\{x\} \cup\left\{y \in X: T_{y}=\emptyset\right\}
$$

Proof. Let $A \in M_{x}$ and $y \in A$ with $y \neq x$. If $T_{y} \neq \emptyset$ there is $D \in M_{y}$. Note that $D \nsubseteq A$ because $D \subseteq A$ would imply that $y \in Z^{A}$, in contradiction with Lemma 13 . Then $x, y \in Z^{A \cup D}$. By IIA and Lemma 12

$$
\left((A \cup D) \backslash Z^{A \cup D}\right) \cup\{x\} \in T_{x} .
$$

So there is $A^{\prime} \in M_{x}$ with

$$
A^{\prime} \subseteq\left((A \cup D) \backslash Z^{A \cup D}\right) \cup\{x\} .
$$

Since $y \in Z^{A \cup D}$, there is $A^{\prime} \in M_{x}$ with $y \notin A^{\prime}$. Hence

$$
\cap\left\{A: A \in M_{x}\right\} .
$$

Remark 19. Lemmas 10-18 imply that each $C \in \mathcal{C}_{2}(n)$ can be identified with an assignment of a collection of subsets $M_{x} \subseteq 2^{X}$ of $X$ to each $x$, and a partition $(Y, Z, W)$ of $X$ such that:

1. $(\forall x \in X)\left(\forall A \in M_{x}\right)(x \in A)$ 


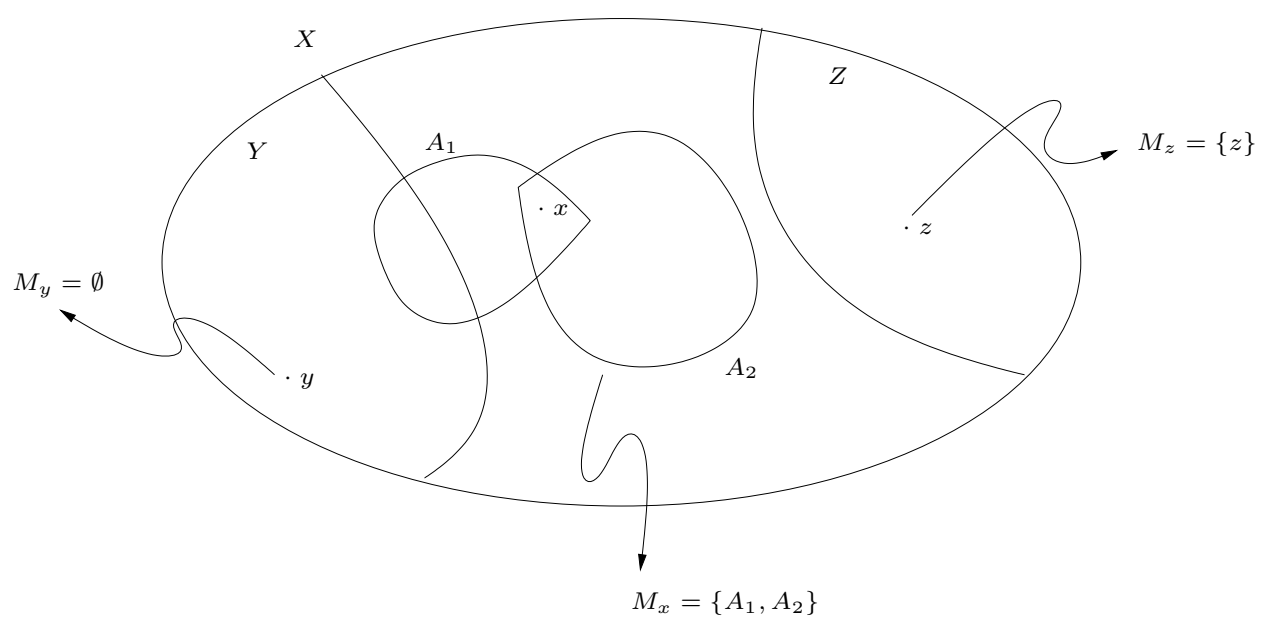

Figure 1: A collection $\left(M_{x}: x \in X\right)$.

2. $(\forall y \in Y)\left(M_{y}=\emptyset\right)$;

3. $(\forall z \in Z)\left(M_{z}=\{\{z\}\}\right)$;

4. $\cup\left\{M_{x}: x \in W\right\}$ is an antichain of subsets of $Y \cup W$;

5. $(\forall x \in W)\left(\{x\} \notin M_{x}\right)$; so $|A| \geq 2$, for all $A \in M_{x}$.

Figure 1 is a sketch of one such collection.

Now fix a partition $(Y, W, Z)$ such that $Y$ and $W$ are nonempty. For each $x \in W$, let $a_{x}$ be some antichain over $Y$. Construct assignment $\left(M_{x}: x \in X\right)$ by letting $M_{x}=$ $\left\{A \cup\{x\}: A \in a_{x}\right\}$, for all $x$ in $W$. Let $M_{y}=\emptyset$ for all $y \in Y$ and let $M_{z}=\{\{z\}\}$ for all $z \in Z$.

Clearly, $\left(M_{x}: x \in X\right)$ defines a system of filters $\left(T_{x}: x \in X\right)$, by $B \in T_{x}$ if and only if there is $A \in M_{x}$ such that $A \subseteq B$. It turns out that

Lemma 20. $\left(T_{x}: x \in X\right)$ satisfies IIA.

Proof. Let $B \subseteq X$. If $x \in Z^{B}$ then there is some $A \in a_{x}$ with $A \cup\{x\} \subseteq B$. Since $A \subseteq Y, T_{y}=\emptyset$ for all $y \in A$. Hence $A \subseteq B \backslash Z^{B}$. Then $\left(B \backslash Z^{B}\right) \cup\{x\} \in T_{x}$.

So $\left(T_{x}\right)$ satisfies IIA by Lemma 12 .

\subsection{Upper and lower bounds}

Let $\mathcal{A}(k)$ be the collection of antichains of the set $\{1,2 \ldots k\}$. Let $\mathcal{B}(k, m)$ be the collection of antichains $a \in \mathcal{A}(k+m)$ such that $(\forall A \in a)(|A| \geq 2)$ and $\cup a \supseteq\{1, \ldots k\}$. 
Let $\eta(k, m)$ be the multinomial coefficient,

$$
\eta(k, m)=\left[\frac{n !}{m ! k !(n-m-k) !}\right]
$$

Lemma 21. $|\mathcal{A}(\lfloor n / 2\rfloor)|^{n-\lfloor n / 2\rfloor} \leq\left|\mathcal{C}_{2}(n)\right|$, and

$$
|\mathcal{A}(n-1)| \leq\left|\mathcal{C}_{2}(n)\right| \leq \sum_{m=1}^{n} \sum_{k=1}^{n-m} \eta(k, m) \theta(k, m) .
$$

Where

$$
\theta(k, m)=\sum_{a \in \mathcal{B}(m, k)}\left\{\sum_{\substack{i_{1}, \ldots i_{k}: \\ i_{1}+\ldots+i_{k}=|a|}} \frac{|a| !}{i_{1} ! \ldots i_{k} !}\right\}
$$

Proof. First, I prove the lower bounds on $\left|\mathcal{C}_{2}(n)\right|$ in Lemma 21. The bounds follow from constructions like those in Lemma 20.

Fix the partition $(Y, W, Z)=(\{1, \ldots\lfloor n / 2\rfloor\},\{\lfloor n / 2\rfloor+1, \ldots n\}, \emptyset)$. For each $x \in W$, let $a_{x} \in A(\lfloor n / 2\rfloor)$ be some antichain. The assignment $M_{x}=\left\{A \cup\{x\}: A \in a_{x}\right\}$ defines

$$
C(A)=\left\{x \in X:\left(\nexists A^{\prime} \in M_{x}\right)\left(A^{\prime} \subseteq A\right)\right\}
$$

Lemma 20 implies that $C$ is substitutable and IIA.

So there are at least as many IIA and substitutable $C$ as maps $A(\lfloor n / 2\rfloor)^{\{\lfloor n / 2\rfloor+1, \ldots n\}}$. This gives the first lower bound in Lemma 21. Letting $(Y, W, Z)=(\{1, \ldots n-1\},\{n\}, \emptyset)$, and reasoning as before, one proves the second lower.

Second, I shall prove the upper bound on $\mathcal{C}_{2}(n)$ in Lemma 21 By Remark 19, there are at most as many $C$ in $\mathcal{C}_{2}(n)$ as partitions $(Y, W, Z)$ of $X$, and assignments of antichains to the elements of $W$ that satisfy the five statements in Remark 19.

Hence, fix integers $m$ and $k$, smaller than $n$. There are $\left(\begin{array}{c}n \\ m\end{array}\right)$ possible choices for $Y$ of cardinality $m$. For each such choice, there are $\left(\begin{array}{c}n-m \\ k\end{array}\right)$ possible choices for $W$ of cardinality $k$. The product $\left(\begin{array}{c}n \\ m\end{array}\right)\left(\begin{array}{c}n-m \\ k\end{array}\right)$ is $\eta(k, m)$. Given $Y$ and $W$, there are at most $\theta(k, m)$ ways of assigning to the elements of $W$ an antichain over $Y \cup W$ that satisfies (4) and (5) in Remark 19. This gives the upper bound on $\mathcal{C}_{2}(n)$.

The upper bound comes from assigning each $x \in A$ some antichain over $X$. The cardinality of these assignments is $|\mathcal{A}(n)|^{n}$

Lemma 22. $\left|\mathcal{C}_{1}(n)\right|=|\mathcal{A}(n-1)|^{n}$ 
Proof. By Lemma 10, $\left|\mathcal{C}_{1}(n)\right|$ equals the number of systems of filters $\left(T_{x}: x \in X\right)$. For each $x$, there are $|\mathcal{A}(n-1)|$ ways of assigning an antichain $M_{x}$ to $x$; each antichain $M_{x}$ is identified with a filter.

Let $S(K, k)$ denote the number of partitions of $\{1, \ldots K\}$ into $k$ sets. $S(K, k)$ is called the Stirling number of the second kind (see e.g. Comtet (1974)).

\section{Lemma 23.}

$$
\sum_{K=0}^{n}\left(\begin{array}{l}
n \\
K
\end{array}\right) \sum_{k=0}^{k}\left(\begin{array}{l}
K \\
k
\end{array}\right)|\mathcal{A}(K)| k ! S\left(\left(\begin{array}{c}
K \\
K / 2\rfloor
\end{array}\right), k\right)
$$

is an upper bound on $\left|\mathcal{C}_{2}(n)\right|$.

Proof. Choose a number $K \in\{0, \ldots n\}$, and choose a subset $(W \cup Y$ ) of $X$ of cardinality $K$. Choose a number $k \in\{0, \ldots K\}$. Choose a subset $W$ of $(W \cup Y$ ) of cardinality $k$.

Choose an antichain $a \in \mathcal{A}(K)$ over $W \cup Y$. An assignment of $a$ to the elements of $W$ gives a partition of $a$. The number of such partitions is $S(|a|,|W|)$, the Stirling Number of the Second Kind. By Sperner's Theorem (Engel (1997)), $|a| \leq\left(\begin{array}{c}K \\ \lfloor K / 2\rfloor\end{array}\right)$. Hence, $S(|a|,|W|) \leq S\left(\left(\begin{array}{c}K \\ \lfloor K / 2\rfloor\end{array}\right), k\right)$.

Now, each partition can be assigned in $k$ ! many ways to the elements of $W$. And there are $|\mathcal{A}(K)|$ antichains over $W \cup Y$. So there are at most $|\mathcal{A}(K)| k ! S\left(\left(\begin{array}{c}K \\ \lfloor K / 2\rfloor\end{array}\right), k\right)$ many assignments of antichain over $W \cup Y$ to the elements of $W$. The stated upper bound follows.

\subsection{Proof of Theorem 2}

First, that $2^{\left(\begin{array}{c}n \\ \lfloor n / 2\rfloor\end{array}\right)} \leq|\mathcal{A}(n)|$ is immediate, since any subset of the collection of subsets

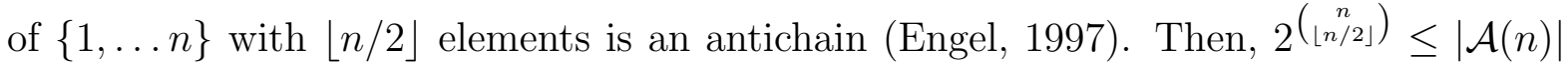
together with the lower bound on $\left|\mathcal{C}_{2}(n)\right|$ in Lemma 21, provide the lower bound on $\left|\mathcal{C}_{2}(n)\right|$ in Theorem 2 .

Second, Kleitman and Markowsky (1975) proved that

$$
|\mathcal{A}(n)| \leq 2^{(1+\kappa \log (n) / n)\left(\begin{array}{c}
n \\
n / 2
\end{array}\right)}
$$

for some constant $\kappa$. Lemma 22 then implies the upper bound on $\left|\mathcal{C}_{1}(n)\right|$, and Lemma 23 the upper bound on $\left|\mathcal{C}_{2}(n)\right|$, in Theorem 2 .

Third, Kleitman (1969) proved that

$$
\log |\mathcal{A}(n)| \sim\left(\begin{array}{c}
n \\
\lfloor n / 2\rfloor
\end{array}\right)
$$

which, by Lemma 22, implies $\log \left|\mathcal{C}_{1}(n)\right| \sim n\left(\begin{array}{c}n-1 \\ \lfloor(n-1) / 2\rfloor\end{array}\right)$. 


\subsection{Note:}

One can use other bounds from the literature on Dedekind's problem, and obtain variations on the results that I present - see Engel (1997) for references to advances on Dedekind's problem.

The best asymptotic estimate for $|\mathcal{A}(n)|$ is due to Korshunov (1981). Clearly, Korshunov's estimate applies to estimating $\left|\mathcal{C}_{1}(n)\right|$ (by Lemma 22).

\subsection{Explanation of the calculations in the Introduction}

The calculation of $\left|\mathcal{C}_{1}(n)\right|$ is from Lemma 22 and the result that $|\mathcal{A}(7)|=2414682040998$ from Comtet (1974). The calculation for $\left|\mathcal{C}_{2}(n)\right|$ follows from the bounds in Theorem 2, but using the known values of $|\mathcal{A}(k)|$. The values $|\mathcal{A}(k)|$, for $k=1 \ldots 7$, are from Comtet, while $|\mathcal{A}(8)|$ is from Wiedemann (1991). Gross (1962) calculates that there are 7087261 preference relations over a set with 9 elements $(8$ elements and $\emptyset$ ). 


\section{Appendix A Rationalizability}

Substitutability and $\beta$ do not imply that $C$ is rationalizable. In fact, substitutability and $\beta$ do not imply that $C$ satisfies IIA. For example: Let $X=\{1,2\}$. Let $C(X)=\emptyset$ and $C(\{i\})=\{i\}$, for $i=1,2$. Then $C$ violates IIA, since $C(X) \subseteq\{1\}$ but $C(\{1\}) \neq C(X)$. It is easy to verify that $C$ satisfies substitutability and $\beta$.

Theorem 24 clears this issue. And it shows another use for the lemmas developed in Section 4.2 .

Theorem 24. $C$ satisfies substitutability, $\beta$ and IIA if and only if $C$ is rationalizable.

Proof. It is immediate that a rationalizable $C$ satisfies substitutability, $\beta$, and IIA.

Let $C$ satisfy substitutability, $\beta$, and IIA. Let $\left(M_{x}: x \in X\right)$ be the system of antichains associated to $C$. Let $\succ$ be the binary relation defined on $X \cup\{\emptyset\}$ by:

- $y \succ x$ if $x \neq y$ and $\{x, y\} \in M_{x}$.

- $\emptyset \succ x$ if $\{x\} \in M_{x} ; x \succ \emptyset$ otherwise.

I shall prove that $\succ$ is transitive. Then I show that $\succ$ rationalizes $C$.

First, I need to prove the following statement:

$$
(\forall A \subseteq X)(\forall B \subseteq X)[(A \subseteq B) \Rightarrow(C(A) \subseteq C(B)) \vee(A \subseteq R(B))]
$$

Let $A \subseteq B$, and $A \nsubseteq R(B)$. By substitutability, $R(A) \subseteq R(B)$; so $A \nsubseteq R(B)$ implies $C(A) \cap C(B) \neq \emptyset$. Now $\beta$ implies $C(A) \subseteq C(B)$.

Second, I shall prove that, $\forall x \in X, \forall A \in M_{x},|A| \leq 2$. Suppose not. Let $x \in X$ and $A \in M_{x}$ with $|A|>2$. Then there is a set $A^{\prime}$ with $\{x\} \subsetneq A^{\prime} \subsetneq A$. Since $A$ is a minimal set from which $x$ is rejected, $x \in C\left(A^{\prime}\right)$. Now, IIA and $x \notin C(A)$, imply $C(A) \nsubseteq C\left(A^{\prime}\right)$. Then, using the statement we proved first, $A^{\prime} \subseteq R(A)$. Let $y \in A^{\prime} \backslash\{x\}$; so $A \in T_{y}$. But then $y \in Z^{A}$ (using the notation introduced before Lemma 12). On the other hand, by Lemma $13,\{x\}=Z^{A}$, for all $A \in M_{x}$. A contradiction.

Third, I prove that $y \succ x$ and $z \succ y$ implies $z \succ x$. Let $A=\{x, y, z\} . y \succ x$ is $\{x, y\} \in M_{x}$, so $A \in T_{x}$; similarly, $A \in T_{y}$. So $\{x, y\} \subseteq Z^{A}$.

Now, $A \backslash Z^{A}=\emptyset$ would imply that $\{x\} \in T_{x}$, as Lemma 12 says that $\{x\} \cup A \backslash Z^{A} \in T_{x}$. But that would contradict that $\{x, y\} \in M_{x}$.

We must have $A \backslash Z^{A} \neq \emptyset$; so $A \backslash Z^{A}=\{z\}$. By Lemma 12, $\{x, z\}=\{x\} \cup A \backslash Z^{A} \in T_{x}$. We know $\{x\} \notin M_{x}$, as $\{x, y\} \in M_{x}$. So $\{x, z\}$ is minimal in $T_{x}$. Hence $\{x, z\} \in M_{x}$. Hence $z \succ x$, and $\succ$ is transitive.

Finally I prove that $\succ$ rationalizes $C$. Fix $A \subseteq X$. Let $x \in C(A)$. Then $\{x\} \notin M_{x}$, as $A \notin T_{x}$. So $x \succ \emptyset$. Let $y \in A$. Then again $A \notin T_{x}$ implies $\{y, x\} \notin M_{x}$. So $y \nsucc x$. 


\section{References}

Aizerman, M., And F. Aleskerov (1995): Theory of Choice, no. 38 in Studies in Mathematical and Managerial Economics. North Holland, Amsterdam.

Austen-Smith, D., and J. S. Banks (1998): Positive Political Theory I : Collective Preference. University of Michigan Press.

BARThÉLÉmy, J.-P. (1980): "An asymptotic equivalent for the number of total preorders on a finite set," Discrete Mathematics, 29(3), 311-313.

Blair, C. (1988): "The Lattice Structure of the Set of Stable Matchings with Multiple Partners," Mathematics of Operations Research, 13(4), 619-628.

Chernoff, H. (1954): "Rational Selection of Decision Functions," Econometrica, 22(4), $422-443$.

Comtet, L. (1974): Advanced Combinatorics. Kluwer Academic Publishers.

Engel, K. (1997): Sperner Theory. Cambridge University Press.

Gross, A. O. (1962): "Preferential Arrangements," The American Mathematical Monthly, 69(1), 4-8.

Kelso, A., And V. Crawford (1982): "Job Matching, Coalition Formation, and Gross Substitutes," Econometrica, 50, 1483-1504.

Kleitman, D. (1969): "On Dedekind's Problem: The Number of Monotone Boolean Functions," Proceedings of the American Mathematical Society, 21(3), 677-682.

Kleitman, D., and G. Markowsky (1975): "On Dedekind's Problem: The Number of Isotone Boolean Functions. II," Transactions of the American Mathematical Society, 213, 373-390.

Korshunov, A. D. (1981): "The number of monotone Boolean functions," Problemy Kibernet, 38, 5-108, In russian, see MathSchiNet entry MR0640855.

Moulin, H. (1991): Axioms of Cooperative Decision Making. Cambridge University Press.

Roth, A., And M. Sotomayor (1990): Two-sided Matching: A Study in GameTheoretic Modelling and Analysis, vol. 18 of Econometric Society Monographs. Cambridge University Press, Cambridge England.

SEgal, I. R. (2003): "The Communication Requirements of Social Choice Rules and Supporting Budget Sets," Mimeo, Stanford University.

Wiedemann, D. (1991): "A computation of the eighth Dedekind number," Order, 8(1), 5-6. 